\title{
Enfermedad celíaca: ¿serología antes de la endoscopía?
}

\author{
Pre-endoscopy serological testing for coeliac disease?
}

\section{Objetivo}

Determinar una herramienta de decisión clínica (HDC) con buen rédito diagnóstico para enfermedad celíaca $(E C)$ y que no incluya realización rutinaria de biopsia duodenal.

Diseño

Estudio antes-después.

\section{Lugar}

Hospital escuela en Sheffield, Inglaterra.

\section{Pacientes}

2000 pacientes consecutivos derivados desde los centros de atención primaria a un centro gastroenterológico en el que se realizaba endoscopía para realizar la misma o para una interconsulta con un gastroenterólogo.

Descripción de los las pruebas diagnósticas y del test de referencia

En primer lugar se analizó una cohorte retrospectiva de pacientes que habían sido derivados para realizar una endoscopía y se creó una HDC que permitiera incrementar la detección de casos de enfermedad celíaca sin necesidad de biopsia duodenal de rutina. La HDC diseñada utilizó la serología (anticuerpos IgA antitransglutaminasa) y la estratificación de los pacientes según los síntomas referidos. Los pacientes fueron clasificados como de "alto riesgo" para enfermedad celíaca si presentaban anemia, pérdida de peso, diarrea o algún síntoma de malabsorción; o de "bajo riesgo" si presentaban cualquier otro síntoma que no incluyera los anteriores. La HDC implicó realizar una biopsia duodenal a todos los pacientes catalogados como "de alto riesgo" y a todos los que tuvieran anticuerpos antitransglutaminasa positivos, independientemente de la clínica. El paso siguiente fue evaluar dicha herramienta en una segunda cohorte prospectiva de pacientes derivados para endos- copía. A todos los pacientes se los clasificó en "alto y bajo riesgo" de acuerdo a su presentación clínica, se les realizó la serología para anticuerpos antitransglutaminasa y una biopsia duodenal de un cuadrante de la segunda porción del duodeno.

\section{Resultados Principales}

La prevalencia de EC en los 2000 pacientes derivados para endoscopia fue de 3,9\% (IC95\% 3,1 a 4,8). La prevalencia en los 739 pacientes catalogados de "alto riesgo" fue 9,6\% (IC95\% 7,7 a 12) y en los 1261 catalogados de "bajo riesgo" fue $0,5 \%$ (IC95\% 0,2\% a 1). En los pacientes que tuvieron anticuerpos antitransglutaminasa negativos la prevalencia fue de $0,4 \%$ (IC95\% 0,2 a 0,7). La sensibilidad de la HDC fue de 100\%.

Tabla 1: características operativas* de la herramienta de decisión clínica para el diagnóstico de enfermedad celíaca sin necesidad de realización rutinaria de una biopsia duodenal.

\begin{tabular}{|c|c|c|c|c|c|c|}
\hline \multirow{2}{*}{$\begin{array}{l}\text { De acuerdo a los } \\
\text { síntomas y los } \\
\text { anticuerpos } \\
\text { antitranglutaminasa }\end{array}$} & \multirow[t]{2}{*}{$\begin{array}{c}\text { Sensibilidad* } \\
\text { e IC95\% }\end{array}$} & \multirow[t]{2}{*}{$\begin{array}{c}\text { Especificidad* } \\
\text { e IC95\% }\end{array}$} & \multicolumn{2}{|c|}{ Valor predictivo* e IC95\% } & \multicolumn{2}{|c|}{$\begin{array}{c}\text { Coeficiente } \\
\text { de } \\
\text { probabilidad* }\end{array}$} \\
\hline & & & Positivo & Negativo & Pos & Neg \\
\hline $\begin{array}{l}\text { Pacientes con } \\
\text { anticuerpos positivos }\end{array}$ & $\begin{array}{c}90,9 \% \\
(82,4 \text { a } 95,5)\end{array}$ & $\begin{array}{c}90,9 \% \\
(89,5 \text { a } 92)\end{array}$ & $\begin{array}{c}28,6 \% \\
(23,3 \text { a } 34,5)\end{array}$ & $\begin{array}{c}99,6 \% \\
(99,2 \text { a } 99,8)\end{array}$ & 9,99 & 0,10 \\
\hline $\begin{array}{l}\text { Pacientes de "alto } \\
\text { riesgo" ó con } \\
\text { anticuerpos positivos }\end{array}$ & $\begin{array}{c}100 \% \\
(5,2 \text { a } 100)\end{array}$ & $\begin{array}{c}60,8 \% \\
(58,6 \text { a } 63)\end{array}$ & $\begin{array}{c}9,3 \% \\
(7,5 \text { a } 11,4 \%)\end{array}$ & $\begin{array}{c}100 \% \\
(99,7 \text { a } 100)\end{array}$ & 2,54 & 0,01 \\
\hline
\end{tabular}

\section{Conclusiones}

La realización de una prueba serológica previa a la endoscopía en combinación con una biopsia sólo en los pacientes clasificados clínicamanete de "alto riesgo" permitió detectar todos los casos de EC.

Palabras claves: enfermedad celíaca, pruebas diagnósticas, biopsia duodenal. Key words: coeliac disease, diagnostic tests, duodenal biopsy.

Fuente de financiamiento/conflicto de interés de los autores: No referida.

\section{Comentario}

La prevalencia de EC en Argentina se estima en uno cada 140 personas'. Clásicamente sólo eran detectados los pacientes que presentaban manifestaciones clínicas claras de malabsorción intestinal, lo que conducía al pedido de una biopsia duodenal. Sin embargo y con la aparición de las determinaciones serológicas, se ha ampliado el espectro de pacientes abarcando también a una población con síntomas más diversos como anemia ( 5 a $12 \%$ de los pacientes con ferropenia son celíacos ${ }^{2}$ ) dermatitis herpetiforme, osteoporosis, enfermedad tiroidea autoinmune, dispepsias tipo "intestino irritable", etc. Es por esta razón que en los últimos años fueron propuestos algoritmos diagnósticos para EC con semejanzas al que hoy resumimos y que incluyen determinaciones serológicas en la toma de decisiones, con el objetivo de evitar biopsiar a todo con sospecha de EC ${ }^{3}$. Un obstáculo a la hora de aplicar esta HDC es que los autores no han reportado explícitamente como se definieron las manifestaciones clínicas que permiten clasificar a los pacientes como de "alto" o de "bajo riesgo" de enfermedad celíaca (primer paso del algoritmo de decisión clínica) a saber: anemia (sólo por los niveles de hemoglobina de acuerdo al sexo, por ma-nifestaciones clínicas de anemia más confirmación del laboratorio, etc.) diarrea (cambio del ritmo evacuatorio, número de deposiciones diarias, etc.), y pérdida de peso (ej. porcentaje de descenso de peso en cierto período, autoreporte por percepción subjetiva de pérdida de peso). Otra limitación de esta validación es que puede sobrestimar el VPP de la HDC ya que fue efectuada sobre una muestra proveniente de un centro de derivación gastroenterológica -población con una prevalencia de enfermedad celícaca de $15,2 \%$ - al que los pacientes habían sido enviados para que se les realizara una endoscopía, lo que sugiere que tenían una alta probabilidad pre-test de la enfermedad, a diferencia de lo que ocurriría en atención primaria, donde la prevalencia es menor a $1 \%$, y donde seguramente el VPP será menor.

\section{Conclusiones del comentador}

Para la práctica clínica es alentador contar con una HDC con una sensibilidad de $100 \%$ para descartar EC en pacientes oligosintomáticos ya que la ausencia de diarrea, pérdida de peso, anemia y anticuerpos antitranglutaminasa (todos estos hallazgos negativos) prácticamente descarta la presencia de EC, lo que conducirá a evitar biopsias innecesarias.

Mercedes Mutchinick y Sergio Terrasa [ Senvicio de Medicina Familiar y Comunitaria del H. Italiano de Buenos Aires. mercedes.mutchinick@ hospitalitaliano.org.ar ] Recibido el 02/01/08 y aceptado el 11/02/08.

Mutchinick M. Enfermedad celíaca: ¿serología antes de la endoscopía? Evid. actual. práct. ambul; 11(1):12, Ene-Feb.2008. Comentado de: Preendoscopy serological testing for coeliac disease: evaluation of a clinical decision tool. Hopper A, Cross S, Hurlstone D y col. BMJ. 2007 Abril 7;334:729-32. PMID: 17383983. Disponible en URL: http://www.bmj.com/cgi/reprint_abr/334/7596/729.pdf (último acceso 05/02/08).

\section{Referencia}

1. De Paula J y col. Diarrea crónica en el adulto. En: Rubisntein A y col. Medicina Familiar y Práctica Ambulatoria. 2da Edición, Buenos Aires, Médica Panamericana, 2006. Pp 1011-1012. 2. Schoj. Evidencia Actualización en la Práctica Ambulatoria - Vol. 3 Número 4: Julio-Agosto 2000. Enfermedad Celíaca en Atención Primaria. http://www.foroaps.org/hitalba-pagina-articulo.php?cod_producto=371

3. Deprati M. Enfermedad Celíaca. Evid. actual. pract. ambul. 2005;8:51-55.Disponible en URL: http://www.foroaps.org/files/gluten.pdf (último acceso 05/02/08). 